\title{
Laminados cerâmicos e preparos minimamente invasivos associados ao clareamento dental
}

Ceramic veneer and minimally invasive procedure associated to dental bleaching

Laminados cerámicos y preparaciones mínimamente invasivas asociadas con el blanqueamiento dental Marina Rodrigues SANTI ${ }^{1}$

Mayara dos Santos NORONHA ${ }^{1}$

Fernanda Rodrigues Borges Amaral GUARATO²

Milena Maria Pierre Santos CALDEIRA ${ }^{2}$

${ }^{1}$ Departamento de Clínica Odontológica, Área de Dentistica. Faculdade de Odontologia de Piracicaba. UNICAMP 13414-903, Piracicaba, SP - Brasil

${ }^{2}$ Departamento de Especialização, Área de Dentistica. Faculdade de Odontologia São Leopoldo Mandic 13045-755 Campinas, SP-Brasil

\section{Resumo}

A indicação de laminados cerâmicos deve ser precedida de um diagnóstico criterioso, onde deve-se estar atento aos passos e aspectos clínicos envolvidos nesse tipo de reabilitação. Este relato de caso tem como objetivo descrever a sequência clínica de uma reabilitação estética dos dentes 11 e 21 empregando laminados cerâmicos de dissilicato de lítio com desgaste dental seletivo minimamente invasivo associado ao clareamento prévio, proporcionando a reprodução das características naturais dos dentes.

Descritores: Clareamento Dental; Cerâmica; Estética Dentária.

\section{Abstract}

The indication of ceramic veeners should be preceded by a careful diagnosis, which should be aware of the steps and aspects related to the type of rehabilitation. This study shows the clinical sequence of an aesthetic rehabilitation of the teeth 11 and 21 with ceramic veneer associated to dental bleaching, reproduction the natural characteristics of the teeth.

Descriptors: Tooth Bleaching; Ceramics; Esthetics, Dental.

\section{Resumen}

La indicación de los laminados cerámicos debe ir precedida de un diagnóstico cuidadoso, donde se debe prestar atención a los pasos y aspectos clínicos que intervienen en este tipo de rehabilitación. Este caso clínico tiene como objetivo describir la secuencia clínica de una rehabilitación estética de los dientes 11 y 21 utilizando laminados cerámicos de disilicato de litio con desgaste dental selectivo mínimamente invasivo asociado a un blanqueamiento previo, proporcionando la reproducción de las características naturales de los dientes.

Descriptores: Blanqueamiento de Dientes; Cerámica; Estética Dental.

\section{INTRODUÇÃO}

A alta exigência e expectativa dos pacientes nos dias atuais, faz com que técnicas minimamente invasivas e mais previsíveis esteticamente sejam desenvolvidas para se atingir a harmonia do sorriso ${ }^{1}$. Dentre as várias opções restauradoras para reabilitações estéticas na região anterior, uma das opções são as facetas laminadas, que destacam-se pela possibilidade de proporcionar um menor desgaste de estruturas dentárias quando comparadas as coroas totais ${ }^{2}$.

Essas facetas indiretas são indicadas quando a queixa estética está relacionada com forma, posição, simetria, textura superficial e cor $^{3}$. Estes procedimentos podem muitas vezes necessitar de modificação de cor prévia, envolvendo nestes casos a associação de agentes clareadores. Dessa forma o profissional deve estar atento aos passos e aspectos clínicos envolvidos nesse tipo de reabilitação ${ }^{4}$.

Dentre as vantagens do uso de laminados cerâmicos, pode-se citar boa estabilidade de cor, fluorescência, longevidade, elevada resistência à compressão, além de apresentar propriedades mecânicas que biomimetizam o esmalte dentário ${ }^{5,6}$.

As cerâmicas reforçadas com dissilicato de lítio apresentam um alto padrão estético, devido ao índice de refração de luz semelhante ao esmalte dental, sem interferência significativa de translucidez, permitindo a possibilidade de reproduzir a naturalidade da estrutura dentária ${ }^{7,8}$. Da mesma forma, o tamanho do cristal e a disposição favorecem maior resistência mecânica e ao desgaste para a restauração ${ }^{8}$.

Sendo assim, para o sucesso clínico, deve-se levar em consideração o tipo de cerâmica a ser utilizado e as características do substrato. Diante desse contexto, este trabalho tem por objetivo relatar o caso de reabilitação estética do sorriso, descrevendo o protocolo clínico de laminados cerâmicos de dissilicato de lítio, com preparos minimamente invasivos associado ao clareamento dental prévio.

\section{CASO CLÍNICO}

Paciente, 51 anos, sexo feminino, compareceu queixando-se de insatisfação em relação à estética do sorriso (Figura 1). Após exame clínico, observou-se um desvio de linha média (Figura 2), além de irregularidades de volume e bordo incisal nos dentes 11 e 21 (Figura 3). Avaliou-se a relação oclusal durante máxima intercuspidação e durante os movimentos de protrusão e lateralidade. Diante da queixa da paciente, elaborou-se o plano de tratamento, propondo restabelecimento estético e funcional do sorriso por meio da associação do clareamento dentário e confecção de laminados cerâmicos com preparos minimamente invasivos.

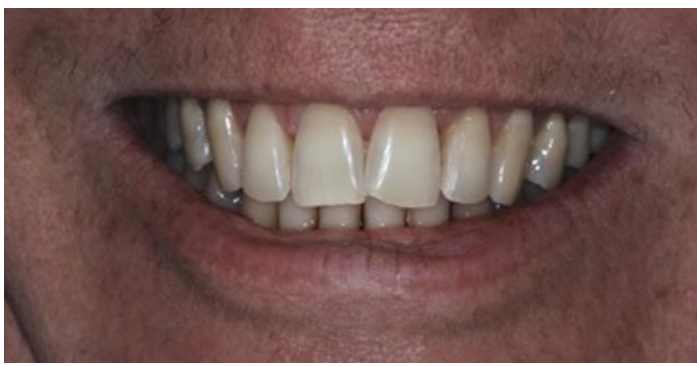

Figura 1: Sorriso inicial. 


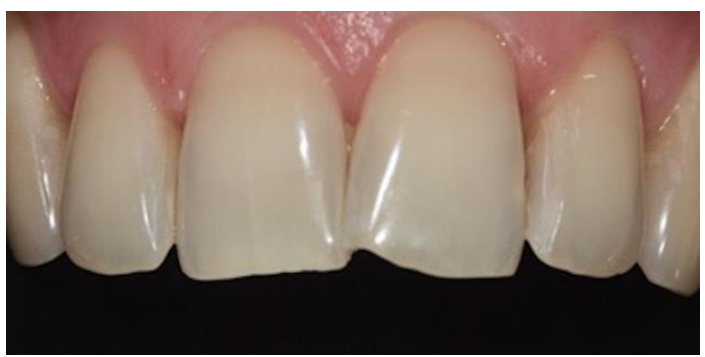

Figura 2: Desvio de linha média e bordos incisais.

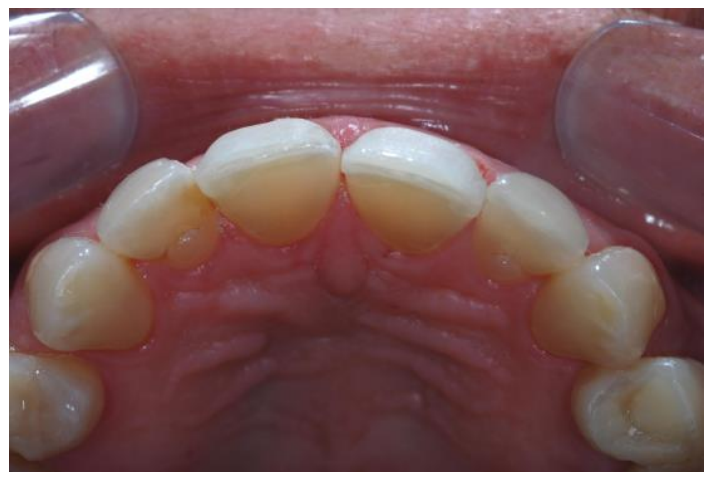

Figura 3: Diferença de volume.

Inicialmente, realizou-se protocolo fotográfico, registro de cor em ficha clínica, moldagem inicial com silicone de adição (Express XT, 3M ESPE, St. Paul, MN, USA) para o enceramento do caso e confecção de moldeira clareadora. De acordo com plano de tratamento, foram realizadas duas aplicações de gel clareador à base de peróxido de hidrogênio a 35\% (Whiteness HP Maxx FGM Produtos Odontológicos, Joinville, $\mathrm{SC}$, Brasil) em sessão única. Em seguida aplicou-se o agente dessensibilizante (KF 2\%, FGM Produtos Odontológicos, Brasil) por 10 minutos. O clareamento de consultório foi associado ao clareamento caseiro durante 15 dias com o gel á base de carbamida 16\% (Whiteness Perfect 16\% - FGM Produtos Odontológicos, Joinville, SC, Brasil). Após o clareamento realizou-se a tomada de cor final das arcadas com escala Vita Classic (Vita, Zahnfabrik, Sackingen, Alemanha) observando-se a redução de um nível de saturação da cor, obtendo como resultado cor A1 (Figura 4).

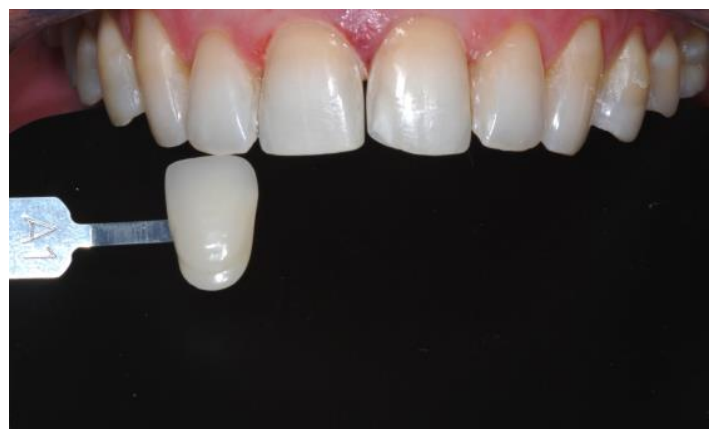

Figura 4: Cor final do substrato dental.

Com a aprovação do enceramento e com o auxílio das guias de silicone (Figura 5), os dentes 11 e 21 receberam preparo minimamente invasivo com remoção apenas das áreas de maior retenção do esmalte com ponta diamantada e pontas de granulação fina e multilaminadas para acabamento. Iniciou-se o preparo com a ponta diamantada cilíndrica de extremidade arredondada no 2243 (KG Sorensen Indústria e Comercio LTDA, São Paulo, SP, Brasil) em três planos: cervical, médio e incisal, realizando um desgaste de $0.4 \mathrm{~mm}$. Removeu-se os pontos de contato para melhor eixo de inserção dos laminados cerâmicos, com a tira de lixa metálica (Flexisaw, Cosmedent, Chicago, IL, EUA). Para refino e polimento dos preparos, utilizou-se as brocas multilaminadas com 12 e 24 lâminas (Ception, Tijucas, SC, Brasil) e disco de feltro (Diamond Flex, FGM) com a pasta de polimento a base de óxido de alumínio Enamelize (Cosmedent).

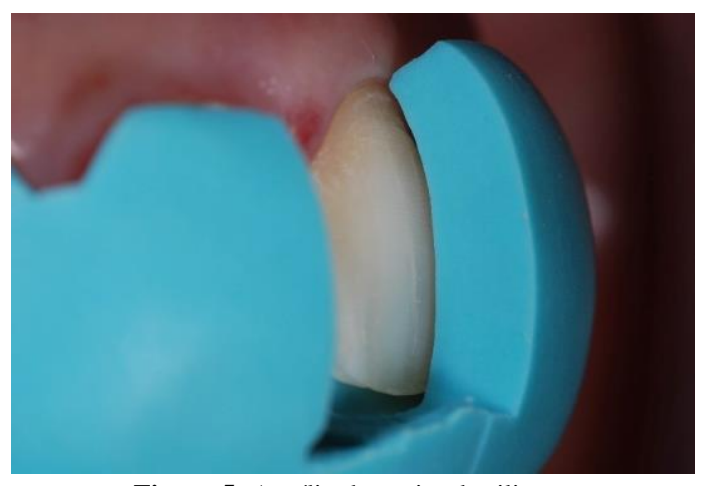

Figura 5: Auxílio das guias de silicone

Após a finalização dos preparos (Figura 6), realizou-se o afastamento gengival com o fio retrator número 0 e 1 (Roeko Retracto, Wilcos do Brasil Indústria e Comércio Ltda, RJ, Brasil) para a moldagem simultânea com silicone de adição (Express XT, 3M ESPE).

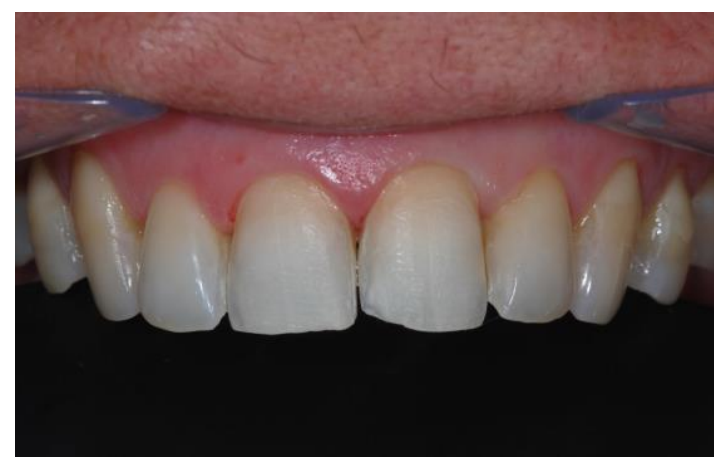

Figura 6: Aspecto final dos preparos

Para finalizar a sessão, aplicou-se o dessensibilizante (KF 0,2\% - FGM) e não houve a necessidade de convecção de mock-up devido à sutileza do preparo. Os laminados cerâmicos foram confeccionados em cerâmica vítrea à base de dissilicato de lítio (IPS E.max, IvoclarVivadent, Schaan, Lichstetein) na cor A2 cervical e A1 terço médio e incisal (Figura 7). Devido a espessura do laminado, optou-se pela cor transparente do cimento resinoso (Universal NX3, Kerr, Collins Avenue Orange, USA). Para condicionamento das peças cerâmicas (IPS E.max, IvoclarVivadent, Schaan, Lichstetein), as superfícies internas foram condicionadas com ácido fluorídrico $10 \%$ (Condac 
porcelana, FGM) durante 20 segundos. Após o tempo de tratamento recomendado, o ácido fluorídrico foi lavado por 60 s e seco e em seguida realizou-se o condicionamento com ácido fosfórico por 60s. As peças foram lavadas e secas novamente. Em seguida, aplicou-se uma camada de silano (Prosil, FGM, Joinville, SC, Brasil) e um breve jato de ar para volatilização do solvente.

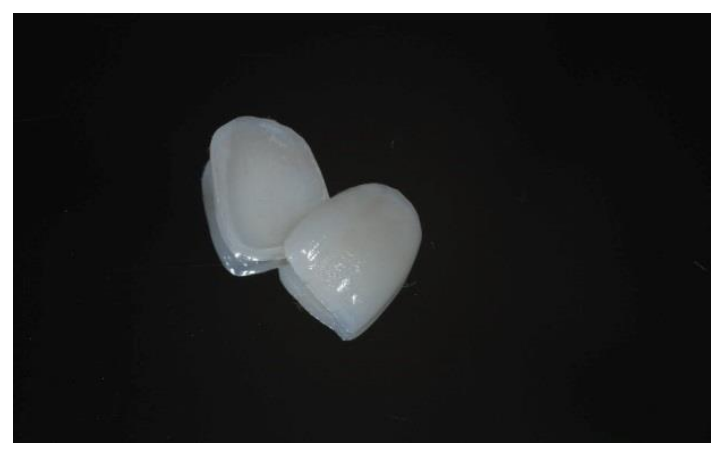

Figura 7: Laminados cerâmicos de dissilicato de lítio.

Para dar início ao procedimento de cimentação, foi realizado o isolamento absoluto modificado (Figura 8).

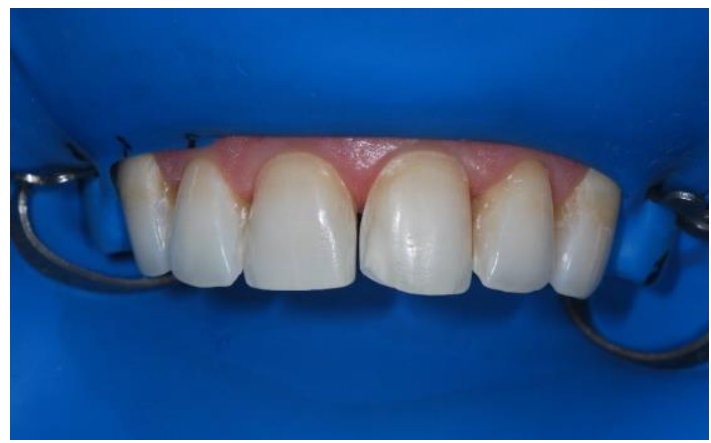

Figura 8: Isolamento absoluto modificado.

$\mathrm{Na}$ estrutura dentária fez-se o condicionamento de todo o preparo com ácido fosfórico a 35\% (Ultra Etch - Ultradent Products, SP, Brasil) por 30 segundos, seguidos da lavagem. Posteriormente foi feita aplicação do sistema adesivo convencional de dois passos (Adper Single Bond 2, $3 \mathrm{M}$ ESPE) e fotoativação por 20 segundos com fotopolimerizador a base de luz LED (Valo Ultradent) com $1400 \mathrm{~mW}$ de potência. Inseriu-se o cimento resinoso de cor transparente (Universal NX3, Kerr), na face interna dos laminados, e posicionou-se sobre os dentes. Os primeiros excessos foram removidos com microbrush e fio dental; realizou-se a fotopolimerização por 40 segundos. Para o acabamento foi utilizada lamina de bisturi $n^{\circ} 12$ na remoção do cimento da região intrasulcular (Figura 9). Para o ajuste oclusal utilizou-se pontas diamantadas 3118F e 3118FF (KG Sorensen) ajustando os contatos em máxima intercuspidação e nos movimentos de guia canina e protrusão. $\mathrm{O}$ polimento nas áreas limitadas aos ajustes foi realizado com pontas impregnadas com silicone (Exa-Cerapol PM, Labordental, SP, Brasil) (Figura 10)

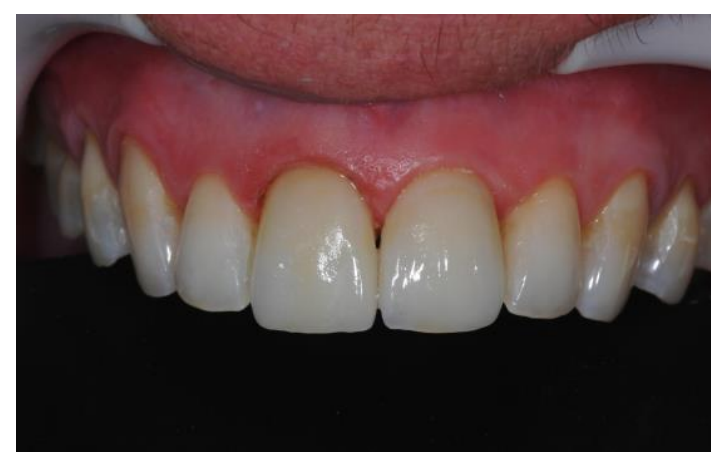

Figura 9: Aspecto final após a cimentação.

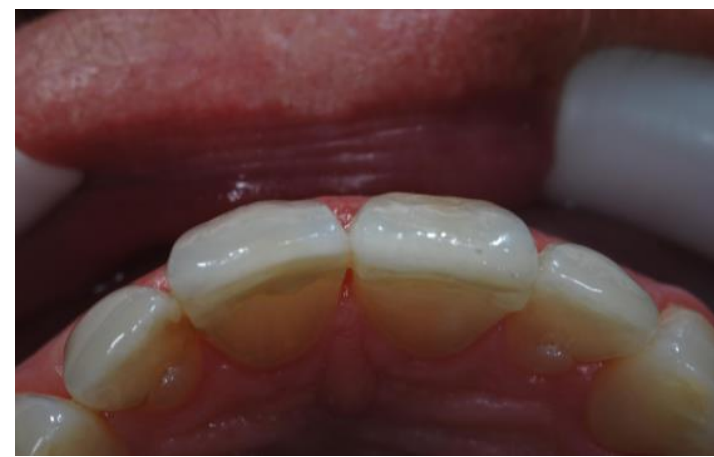

Figura 10: Correção de volume dos dentes 11 e 21.

$O$ resultado final demonstra o aspecto do sorriso inicial e final após a reabilitação que modificou significativamente a harmonia estéticofuncional do sorriso. (Figura 11)

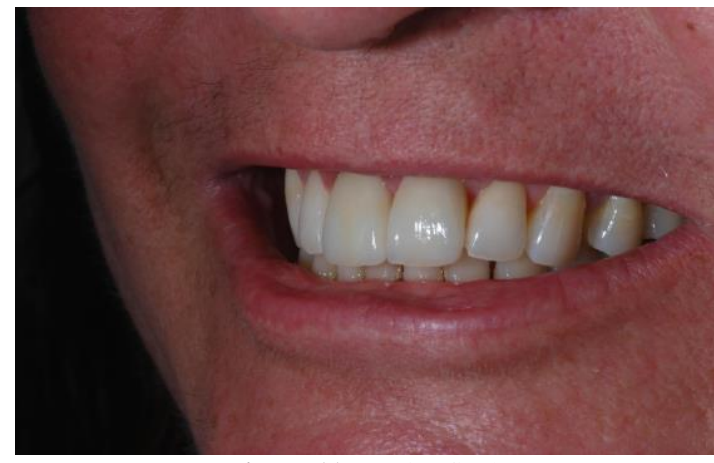

Figura 11: Sorriso final.

\section{CONSIDERAÇÕES FINAIS}

Ser conservador não está relacionado com a espessura do laminado cerâmico, ser conservador é saber escolher a abordagem de maior efetividade com o menor custo das estruturas dentárias sadias.

\section{REFERENCIAS}

1. D'Arcangelo C, De Angelis F, Vadini M, D'Amario M. Clinical evaluation on porcelain laminate veneers bonded with light-cured composite: results up to 7 years. Clin Oral Investig. 2012;16(4):1071-79.

2. Benetti AR, Miranda CB, Amore R, Pagani C. Facetas indiretas em porcelana-alternativa estética. J Bras Dent Estét. 2003;2(7):186-94.

3. Teixeira HM, Nascimento ABL, Emerrenciano M. Reabilitação da Estética com Facetas Indiretas de Porcelana. J Bras Dent Estét. 2003;2(7):219-23.

4. Jarad FD, Griffiths CE, Jaffri M, Adeyemi AA, 
Youngson CC. The effect of bleaching, varying the shade or thickness of composite veneers on final colour: an in vitro study. J Dent. 2008;36(7):554-59.

5. Lowe E. Achieving total smile enhancement through tooth whitening and placement of a single anterior veneer restoration. Dent Today. 2001;20(12):44-8

6. Fradeani M, Redemagni M, Corrado M. Porcelain laminate veneers: 6- to 12-year clinical evaluation--a retrospective study. Int $\mathbf{J}$ Periodontics Restorative Dent. 2005;25(1):9-17.

7. Aquino APT, Cardoso PC, Rodrigues MB, Takano AE, Porfírio W. Facetas de porcelana: solução estética e funcional. Clin int $\mathrm{j}$ braz dent. 2009;5(2):142-52.

8. Higashi C, Reggiani RD, Kina S, Scopin O, Hirata R. Cerâmicas em Dentes Anteriores Parte I. Indicações Clínicas dos sistemas cerâmicos. Clin int $\mathrm{j}$ braz dent. 2006;2(1):22-31.

9. Kina S, Brugrera A. Inivisível: restaurações estéticas cerâmicas. Maringá: Dental Press; 2007.

\section{CONFLITO DE INTERESSES}

Os autores declaram não haver conflitos de interesse.

\section{AUTOR PARA CORRESPONDÊNCIA}

\section{Marina Rodrigues Santi \\ Av. Limeira, 901 - Areião, 13414-903 Piracicaba - SP, Brasil email: marina.rsanti@gmail.com}

Submetido em 12/08/2019

Aceito em 20/04/2020 